This item was submitted to Loughborough's Research Repository by the author.

Items in Figshare are protected by copyright, with all rights reserved, unless otherwise indicated.

\title{
Contact force estimation in the railway vehicle wheel-rail interface
}

PLEASE CITE THE PUBLISHED VERSION

http://dx.doi.org/10.3182/20110828-6-IT-1002.02904

\section{PUBLISHER}

(c) International Federation of Automatic Control

\section{VERSION}

VoR (Version of Record)

\section{LICENCE}

CC BY-NC-ND 4.0

\section{REPOSITORY RECORD}

Ward, Christopher P., Roger M. Goodall, and Roger Dixon. 2019. "Contact Force Estimation in the Railway Vehicle Wheel-rail Interface”. figshare. https://hdl.handle.net/2134/8773. 
This item was submitted to Loughborough's Institutional Repository (https://dspace.lboro.ac.uk/) by the author and is made available under the following Creative Commons Licence conditions.

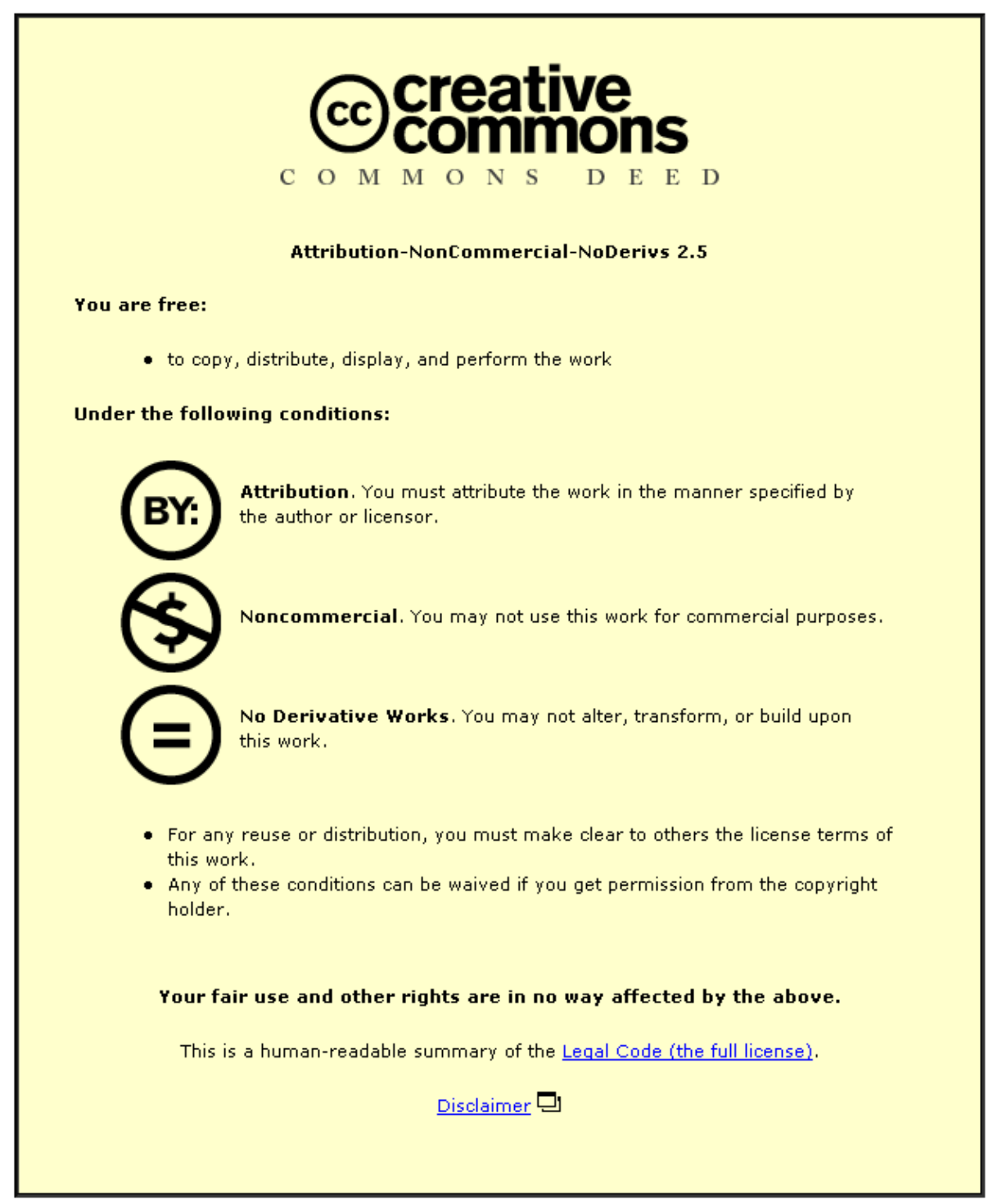

For the full text of this licence, please go to: http://creativecommons.org/licenses/by-nc-nd/2.5/ 


\title{
Contact Force Estimation in the Railway Vehicle Wheel-Rail Interface
}

\author{
Christopher P. Ward ${ }^{*}$ Roger M. Goodall* Roger Dixon* \\ * Department of Electronic and Electrical Engineering, Loughborough \\ University, Loughborough, Leicestershire, LE11 3TU, UK(e-mail: \\ c.p.ward@lboro.ac.uk; r.m.goodall@lboro.ac.uk; r.dixon@lboro.ac.uk)
}

\begin{abstract}
Increased patronage of railways in the UK in the past 20 years has put demands on rolling stock to operate at peak availability with reduced time available for maintenance. One possible tool to enable this is the use of real time fault detection and diagnosis on board railway vehicles to detect faulty components and provide information about the current running condition of the system. This paper discusses the development of one such technique for the estimation of creep forces of the wheel-rail contact. Real time knowledge of which could be used to predict wear of the wheel tread and rail head, predict the formation of rolling contact fatigue, and identify any areas of low adhesion present on the network. The paper covers development of a full vehicle nonlinear contact mechanics model, development of the Kalman-Bucy filter estimation technique and how the technique will be developed and validated in the future.
\end{abstract}

Keywords: Accelerometers, Fault Diagnosis/Detection, Kalman Filters, Nonlinear Systems, Railways, Vehicle Dynamics

\section{INTRODUCTION}

The railway industry in the United Kingdom has seen something of a renaissance in the past 20 years with annual increases in passenger numbers and tonnage of freight hauled, putting demands on rolling stock to be available for a greater proportion of the time. This results in a reduction in maintenance availability and a requirement to better understand the current running conditions so that timetables can be tailored to the peak operating capability of the current rolling stock.

A key tool in improving the capability of the system is the use of condition monitoring systems on board railway vehicles to detect faults and estimate running conditions in real time. The railway industry is starting the transition to this philosophy with systems such as the Bombardier ORBITA, Bombardier (2010). In academia many condition monitoring schemes have been investigated, such as: condition monitoring of suspension components, $\mathrm{Li}$ et al. (2006); wheel-rail profile estimation, Ward et al. (2010a); wheel speed measurement, Mei and Li (2008) and creep force detection, Charles et al. (2008).

This paper highlights development of an estimation technique to determine, in real time, the creep forces of the wheel rail interface. Estimation of these forces has potentially many benefits as currently this can only be measured with specially instrumented trains, the technology from which would be prohibitively expensive to install on all service vehicles. Creep forces fundamentally provide the guidance mechanism of the wheelset system by dissipating energy in the contact area, Wickens (2003). As such creep forces and moments are a key part of the mechanism for wheel and rail head wear, the generation of rolling contact fatigue of the rail head and the amount of adhesion that is present for traction and braking. Force estimation in real time on each vehicle on the network will provide information by which it is possible to detect: the wear of the system to provide more targeted maintenance regimes; and areas of low adhesion so that mitigation such as rail head cleaning and treatment could be better deployed, Vasic et al. (2003). This paper is focused upon the estimation of creep forces, detection of the issues highlighted using the data provided by estimation is the subject of on-going research.

A number of ideas have been proposed to detect the running condition of the wheel-rail interface that use low cost inertial sensing mounted on the vehicle and advanced processing, such as: multiple Kalman filters to estimate creep coefficients, Hussain and Mei (2010); inverse modelling for the estimation of creep forces, Xia et al. (2008); and as first proposed in Charles et al. (2008) and further developed here, a Kalman-Bucy filter estimation of linearised creep forces, initially aimed at detecting local adhesion conditions. This paper therefore covers the nonlinear contact mechanics modelling of a full bodied vehicle, with two bogies and four wheelset. It then covers the Kalman-Bucy filtering estimation technique, with preliminary simulation results shown. Finally it discusses how the technique will be developed in the future and plans for experimental validation.

\section{SYSTEM MODEL}

Previous studies into creep force estimation have looked at a half vehicle models only, Charles et al. (2008), Ward et al. (2010b), where just one bogie system is considered, with the vehicle body constrained in yaw. This model has been sufficient for formative theoretical studies, however it demonstrated some discrepancies in the observability 
of various creep forces at different parts on the vehicle system, with the estimated lateral creep force of the trailing wheelset giving best correlation to the modelled creep force. Therefore this study is looking into the best candidate creep forces for detection on a more representative system.

The current study considers a vehicle model with a full length body no longer constrained in yaw, plus two bogies with the accompanying four wheelsets, Figure 1. As with previous studies this model only considers the lateral and yaw directions, as the longitudinal and vertical effects can be satisfactorily neglected, Wickens (2003).

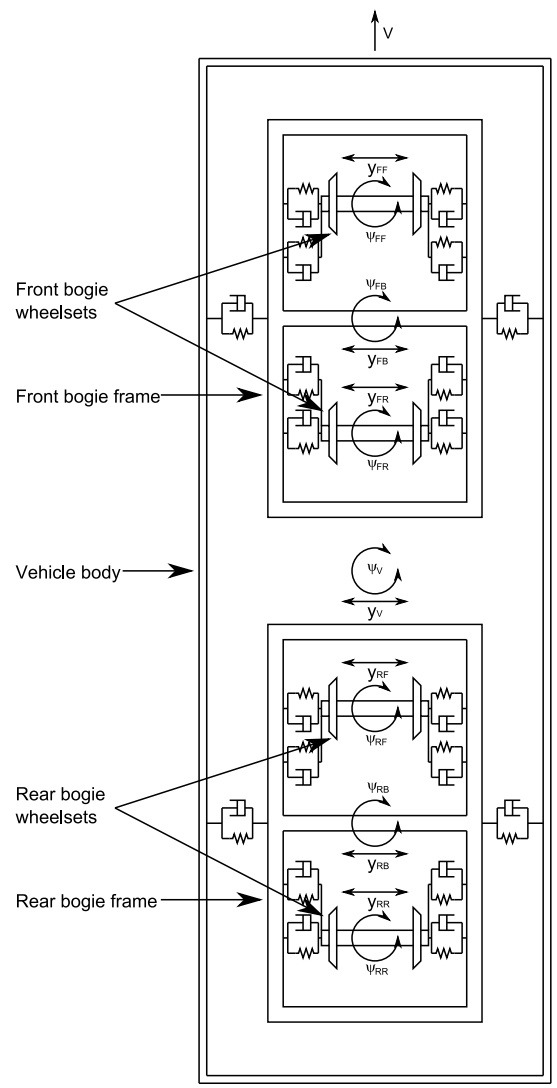

Fig. 1. Vehicle system plan view model

A detailed description of the development of railway vehicle dynamics can be found in Garg and Dukkipati (1984), with the rigid body dynamics for the simulation model given below by equations 1 to 14. These equations encompass lateral and yaw dynamics for the four wheelsets, two bogies and the vehicle body.

$$
\begin{aligned}
m_{F F} \ddot{y}_{F F}= & F_{L y F F}+F_{R y F F}+F_{s y F F}+F_{g F F} \\
I_{F F} \ddot{\psi}_{F F} & =F_{L y F F} R_{L x F F}-F_{L x F F} R_{L y F F} \\
& +F_{R y F F} R_{R x F F}-F_{R x F F} R_{R y F F} \\
& +M_{s \psi F F}+M_{g F F} \\
m_{F R} \ddot{y}_{F R}= & F_{L y F R}+F_{R y F R}+F_{s y F R}+F_{g F R} \\
I_{F R} \ddot{\psi}_{F R} & =F_{L y F R} R_{L x F R}-F_{L x F R} R_{L y F R} \\
& +F_{R y F R} R_{R x F R}-F_{R x F R} R_{R y F R} \\
& +M_{s \psi F R}+M_{g F R} \\
m_{R F} \ddot{y}_{R F}= & F_{L y R F}+F_{R y R F}+F_{s y R F}+F_{g R F}
\end{aligned}
$$

$$
\begin{gathered}
I_{R F} \ddot{\psi}_{R F}=F_{L y R F} R_{L x R F}-F_{L x R F} R_{L y R F} \\
+F_{R y R F} R_{R x R F}-F_{R x R F} R_{R y R F} \\
+M_{s \psi R F}+M_{g R F} \\
m_{R R} \ddot{y}_{R R}=F_{L y R R}+F_{R y R R}+F_{s y R R}+F_{g R R} \\
I_{R R} \ddot{\psi}_{R R}=F_{L y R R} R_{L x R R}-F_{L x R R} R_{L y R R} \\
+F_{R y R R} R_{R x R R}-F_{R x R R} R_{R y R R} \\
+M_{s \psi R R}+M_{g R R} \\
m_{F B} \ddot{y}_{F B}=-\left(F_{s y F F}+F_{s y F R}+F_{s y V F}\right) \\
\ddot{I}_{F B} \ddot{\psi}_{F B}=-\left(M_{s \psi F F}+M_{s \psi F R}+M_{s y V F}\right. \\
\left.\quad+L\left(F_{s y F F}-F_{s y F R}\right)\right) \\
m_{R B} \ddot{y}_{R B}=-\left(F_{s y R F}+F_{s y R R}+F_{s y V R}\right) \\
I_{R B} \ddot{\psi}_{R B}=-\left(M_{s \psi R F}+M_{s \psi R R}+M_{s y V R}\right. \\
\left.\quad+L\left(F_{s y R F}-F_{s y R R}\right)\right) \\
m_{V} \ddot{y}_{V}=F_{s y V F}+F_{s y V R} \\
I_{V} \ddot{\psi}_{V}=M_{s \psi V F}+M_{s \psi V R}
\end{gathered}
$$

where $F_{i j k l}, R_{i j k l}, M_{i \psi k l}$ are the forces (creep, gravitational and suspension), positions and moments, $m_{k l}$ is the mass, $I_{k l}$ is the moment of inertia, $y_{k l}$ is the lateral position, $\psi_{k l}$ is the yaw angle; where $i=L$ (eft), $R$ (ight), $s$ (uspension); $j=x$ (longitudinal), $y$ (lateral); $k=F$ (ront bogie), $R$ (rear bogie), $V$ (vehicle); $l=F$ (ront wheelset), $R$ (rear wheelset), $B$ (ogie)

The accompanying suspension forces and moments (for small angles) for the primary and secondary suspension are given by equations 15 to 26 .

$$
\begin{gathered}
F_{s y F F}=k_{y 1} y_{B F}+k_{y 1} L \psi_{B F}-k_{y 1} y_{F F} \\
+f_{y 1} \dot{y}_{B F}+f_{y 1} L \dot{\psi}_{B F}-f_{y 1} \dot{y}_{F F} \\
M_{s \psi F F}=k_{\psi 1}\left(\psi_{B F}-\psi_{F F}\right)+f_{\psi 1}\left(\dot{\psi}_{B F}-\dot{\psi}_{F F}\right) \\
F_{s y F R}=k_{y 1} y_{B F}-k_{y 1} L \psi_{B F}-k_{y 1} y_{F R} \\
+f_{y 1} \dot{y}_{B F}-f_{y 1} L \dot{\psi}_{B F}-f_{y 1} \dot{y}_{F R} \\
M_{s \psi F R}=k_{\psi 1}\left(\psi_{B F}-\psi_{F R}\right)+f_{\psi 1}\left(\dot{\psi}_{B F}-\dot{\psi}_{F R}\right) \\
F_{s y R F}=k_{y 1} y_{B R}+k_{y 1} L \psi_{B R}-k_{y 1} y_{R F} \\
+f_{y 1} \dot{y}_{B R}+f_{y 1} L \dot{\psi}_{B R}-f_{y 1} \dot{y}_{R F} \\
M_{s \psi R F}=k_{\psi 1}\left(\psi_{B R}-\psi_{R F}\right)+f_{\psi 1}\left(\dot{\psi}_{B R}-\dot{\psi}_{R F}\right) \\
F_{s y R R}=k_{y 1} y_{B R}-k_{y 1} L \psi_{B R}-k_{y 1} y_{R R} \\
+f_{y 1} \dot{y}_{B R}-f_{y 1} L \dot{\psi}_{B R}-f_{y 1} \dot{y}_{R R} \\
M_{s \psi R R}=k_{\psi 1}\left(\psi_{B R}-\psi_{R R}\right)+f_{\psi 1}\left(\dot{\psi}_{B R}-\dot{\psi}_{R R}\right) \\
F_{s y V F}=-k_{y 2} y_{V}-f_{y 2} \dot{y}_{V}+k_{y 2} y_{B F}+f_{y 2} \dot{y}_{B F} \\
-k_{y 2} c \psi_{V}-f_{y 2} c \dot{\psi}_{V} \\
M_{s \psi V F}=-k_{y 2} c^{2} \psi_{V}-f_{y 2} c^{2} \dot{\psi}_{V}+k_{y 2} c y_{V}+f_{y 2} c \dot{y}_{V} \\
-k_{y 2} c y_{B F}-f_{y 2} c \dot{y}_{B F} \\
F_{s y V R}=-k_{y 2} y_{V}-f_{y 2} \dot{y}_{V}+k_{y 2} y_{B R}+f_{y 2} \dot{y}_{B R} \\
+k_{y 2} c \psi_{V}+f_{y 2} c \dot{\psi}_{V} \\
M_{s \psi V R}=-k_{y 2} c^{2} \psi_{V}-f_{y 2} c^{2} \dot{\psi}_{V}-k_{y 2} c y_{V}-f_{y 2} c \dot{y}_{V} \\
-k_{y 2} c y_{B R}-f_{y 2} c \dot{y}_{B R}
\end{gathered}
$$

where $k_{m n}$ and $f_{m n}$ are the suspension stiffness and damper coefficients; with $m=y$ (lateral) or $\psi$ (yaw); $n=$ 1(primary suspension), 2(secondary suspension).

Creep forces fundamentally provide the guidance mechanism for the wheelsets. These forces are generated in reaction to the creeps (or slips) in the rolling contact of the wheel-rail interface in normal running, these are relative 
velocities of the wheel and the rail in the contact area and are defined as

$$
s_{i}=\frac{w_{i}}{V}, i=x, y
$$

where $V$ is the forward velocity of the wheelset, $w_{i}$ is the creep (slip) velocity in the relevant direction (where $x$ is longitudinal direction and $y$ is lateral direction), where this is defined as

$$
w_{i}=V_{w}-V_{r}, i=x, y
$$

where $V_{w}$ is the velocity of the wheel through the contact patch, and $V_{r}$ is the velocity of the rail through the contact patch. Creep generation is a highly nonlinear process, however effects of hysteresis can be ignored due to this being a single direction rolling contact.

Normal practice for wheel-rail contact modelling is to linearise the creep forces generated in the model based upon Kalker coefficients, Kalker (1967). Due to the importance here of modelling the non-linear adhesion characteristics up to and beyond the creep saturation, use is made of the contact force model developed in Polach (2005). This is essentially a practical curve fitting mechanism, where the creep force (excluding spin effects) are calculated as

$$
F=\frac{2 Q \mu}{\pi}\left(\frac{\epsilon}{1+\epsilon^{2}}+\arctan \epsilon\right)
$$

where $Q$ is the wheel load, with

$$
\epsilon=\frac{2}{3} \frac{C \pi a^{2} b}{Q \mu} s
$$

where $C$ is the proportionality coefficient of the contact sheer stiffness $\left(\mathrm{N} / \mathrm{m}^{3}\right)$. Kalker coefficients can be used for this purpose, where for the longitudinal direction

$$
\epsilon_{x}=\frac{1}{4} \frac{G \pi a b c_{11}}{Q \mu} s_{x}
$$

where $s_{x}$ is the longitudinal component of the total creep

$$
s=\sqrt{s_{x}^{2}+s_{y}^{2}}
$$

The forces $F_{x}, F_{y}$ in the longitudinal and lateral directions are

$$
F_{i}=F \frac{s_{i}}{s}, i=x, y
$$

and the adhesion coefficients

$$
f_{i}=\frac{F_{i}}{Q}, i=x, y
$$

The friction coefficients rely upon the slip velocity, where

$$
\mu=\mu_{0}\left[(1-A) e^{-B w}+A\right]
$$

$A$ is the ratio of limit friction coefficient at infinity slip velocity $\mu_{\infty}$ to the maximum friction coefficient $\mu_{0}$

$$
A=\frac{\mu_{\infty}}{\mu_{0}}
$$

For large creep applications the force is worked out using reduction factors, $k_{A}$ in the area of adhesion and $k_{S}$ in the area of slip, as

$$
F=\frac{2 Q \mu}{\pi}\left(\frac{k_{A} \epsilon}{1+\left(k_{A} \epsilon\right)^{2}}+\arctan \left(k_{S} \epsilon\right)\right), k_{S} \leq k_{A} \leq 1
$$

where

$$
k=\frac{k_{A}+k_{S}}{2}
$$

Experimentation has shown that, contrary to expectation from theoretical models such as that of Kalker, Kalker
(1967), the initial slope of the creep curve varies with different adhesion levels, Pearce and Rose (1985), Harrison and McCanney (2002). Four levels of adhesion are defined in this study as dry, wet, low and very low conditions. The accompanying constants are given in Table 1 and the creep curves are given in Figure 2. This varying slope means that different adhesion levels should be feasibly detected without the system becoming saturated. The effect of varying the adhesion levels on the running system is shown in Figure 3. This shows the sum of the lateral creep forces and gravitational stiffnesses for the rear bogies front wheelset and demonstrates that for the same system disturbance (i.e. the lateral position of the track), the creep forces generated reduces as the friction levels reduce, meaning that detection of changes of adhesion level is feasible in practice.

\begin{tabular}{|c|c|c|c|c|}
\hline Model parameter & Dry & Wet & Low & Very Low \\
\hline$k_{A}$ & 1.00 & 1.00 & 1.00 & 1.00 \\
$k_{s}$ & 0.40 & 0.40 & 0.40 & 0.40 \\
$\mu_{0}$ & 0.55 & 0.30 & 0.06 & 0.03 \\
$A$ & 0.40 & 0.40 & 0.40 & 0.40 \\
$B$ & 0.60 & 0.20 & 0.20 & 0.10 \\
\hline
\end{tabular}

Table 1. Polach contact model parameters

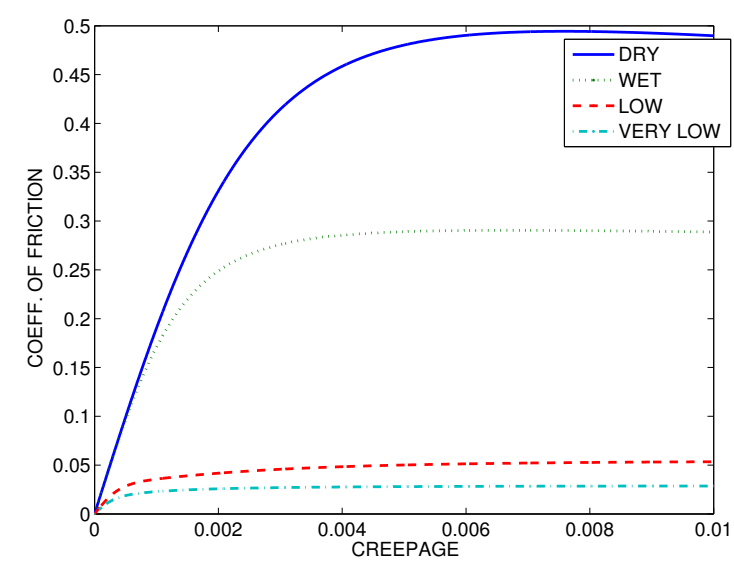

Fig. 2. Creep curves for varying adhesion conditions

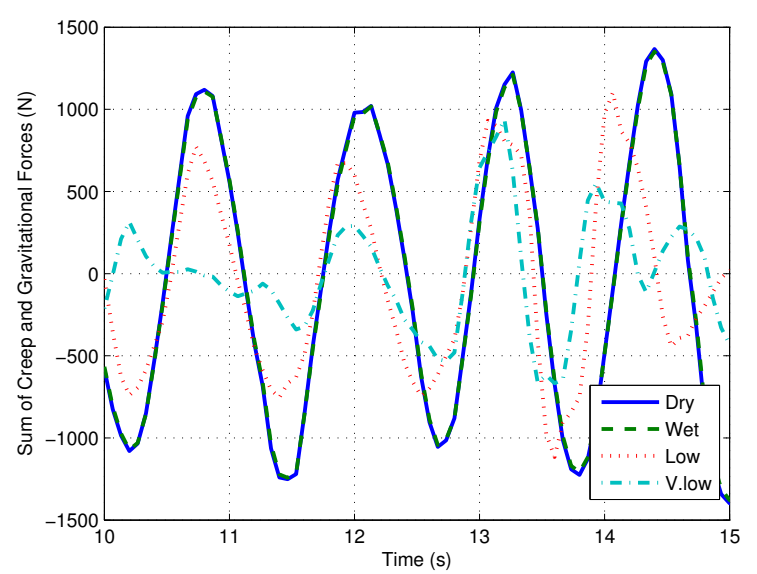

Fig. 3. Lateral creep forces for varying adhesion conditions 


\section{CREEP FORCE ESTIMATION TECHNIQUE}

This study applies a Kalman-Bucy filter technique (Kalman (1960)) as developed for the half vehicle model in Charles et al. (2008), to the full vehicle model of the previous section. As mentioned earlier this method attempts to estimate the total reactive creep and gravitational forces generated at the wheel rail interface. The size of these creep forces can then be analysed to determine the level of adhesion present, and the impact of the rail vehicle upon the track infrastructure.

The Kalman-Bucy filter model uses simplified versions of the system equations 1 to 8 . As previous studies highlighted, the detection technique used here can not differentiate between left and right creep forces, and the gravitational stiffness present. Therefore these are brought together as one state to be estimated, these are now

$$
\begin{gathered}
m_{F F} \ddot{y}_{F F}=F_{F F}+F_{s y F F} \\
I_{F F} \ddot{\psi}_{F F}=M_{F F}+M_{s \psi F F} \\
m_{F R} \ddot{y}_{F R}=F_{F R}+F_{s y F R} \\
I_{F R} \ddot{\psi}_{F R}=M_{F R}+M_{s \psi F R} \\
m_{R F} \ddot{y}_{R F}=F_{R F}+F_{s y R F} \\
I_{R F} \ddot{\psi}_{R F}=M_{R F}+M_{s \psi R F} \\
m_{R R} \ddot{y}_{R R}=F_{R R}+F_{s y R R} \\
I_{R R} \ddot{\psi}_{R R}=M_{R R}+M_{s \psi R R}
\end{gathered}
$$

where for the purposes of the filter, the following assumptions are made

$$
\begin{gathered}
\dot{F}_{F F}=\dot{F}_{F R}=\dot{F}_{R F}=\dot{F}_{R R}=0 \\
\dot{M}_{F F}=\dot{M}_{F R}=\dot{M}_{R F}=\dot{M}_{R R}=0
\end{gathered}
$$

For this proof of concept work it is assumed that all of the states can be measured. Practically this will not be possible, so later stages of the work will look into reducing the number of states to be measured, and gauging how many can be estimated without detrimentally affecting the creep force estimation quality. The Kalman-Bucy filter, Grewal and Andrews (2001), is a very well known state space method, where the state equation is defined as

$$
\dot{x}=A_{k} x+B_{k} u+z
$$

where $x$ is the state vector, $\dot{x}$ is the rate of change of the state vector, $z$ is the Gaussian noise source on each of the state vectors, $A_{k}$ is the state matrix and $B_{k}$ is the input matrix. The output equation for the system is defined as

$$
y=C_{k} x+D_{k} u+v
$$

where $y$ is the output vector, $v$ is the Gaussian noise on the output vector, $C_{k}$ is the output matrix and $D_{k}$ is the input matrix.

Design choices are made by selecting covariance matrices of the state and the input. These define how much noise is present in the system, therefore affecting the output of the system due to the model measurements not exactly matching the system.

The filter algorithm can be split into two sections. The first section calculates how much to adapt the filter to changes in the system.

$$
\begin{gathered}
K=P C_{K}^{T} R^{-1} \\
\dot{P}=A_{k} P+P A_{k}^{T}-K R K^{T}+Q
\end{gathered}
$$

where $K$ is the 'Kalman gain', $P$ is the error covariance which in this continuous case the initial conditions of which require setting.

The second part is updating the estimates. The estimated state and output are then calculated simultaneously as

$$
\begin{gathered}
\hat{y}=C_{k} \hat{x}+D_{k} u \\
\dot{\hat{x}}=A_{k} \hat{x}+B_{k} u+K(y-\hat{y})
\end{gathered}
$$

where $\hat{y}$ is the estimated output and $\hat{x}$ is the estimated state.

In this example, it is assumed that there is no system input, and the filter becomes output only, therefore $C_{k}=$ $D_{k}=0$. Where the state vector is defined as

$$
\begin{aligned}
x=[ & y_{F F} \dot{y}_{F F} \psi_{F F} \dot{\psi}_{F F} y_{F R} \dot{y}_{F R} \psi_{F R} \dot{\psi}_{F R} \cdots \\
& \cdots y_{B F} \dot{y}_{B F} \psi_{B F} \dot{\psi}_{B F} y_{R F} \dot{y}_{R F} \cdots \\
& \cdots \psi_{R F} \dot{\psi}_{R F} y_{R R} \dot{y}_{R R} \psi_{R R} \dot{\psi}_{R R} \cdots \\
& \cdots y_{B R} \dot{y}_{B R} \psi_{B R} \dot{\psi}_{B R} y_{V} \dot{y}_{V} \cdots \\
& \cdots \psi_{V} \dot{\psi}_{V} F_{F F} F_{F R} F_{R F} F_{R R} \cdots \\
& \left.\cdots M_{F F} M_{F R} M_{R F} M_{R R}\right]^{T}
\end{aligned}
$$

The primary tuning parameter here is the $Q$ matrix, defined as a diagonal matrix, where

$$
\begin{gathered}
Q=\operatorname{diag}[11111111111111 \cdots \\
\cdots 11111111111111 \cdots \\
\left.\cdots 1 e^{9} 1 e^{9} 1 e^{9} 1 e^{9} 1 e^{9} 1 e^{9} 1 e^{9} 1 e^{9}\right]
\end{gathered}
$$

The high values associated with the last eight positions in the matrix assign uncertainty to the assumptions of equations 47 and 48, allowing the filter to adapt the state estimates to the creep force levels.

Figure 4 show results of the filter output compared to the modelled total lateral creep forces for the rear wheelset of the front bogie, by inspection this shows good correlation for the specific estimate. The simulation parameters are for dry friction and the model velocity is $20 \mathrm{~m} / \mathrm{s}$, with suspension parameters given in Table 2. The coefficient of determination $\left(R^{2}\right)$ values for the four lateral creep forces from the simulations with different levels of adhesion are shown in Table 3, where this is calculated as

$$
R^{2}=1-\frac{\sigma^{2}(\epsilon(t))}{\sigma^{2}(y(t))}
$$

where $\sigma^{2}(\epsilon(t))$ is the variance of the residuals and $\sigma^{2}(y(t))$ is the variance of the output, Ljung (1999). This shows that there is some reduction in the quality of the creep force estimation as the friction level reduces. This is due to the nonlinear effects of the creep force saturation becoming more prominent as the adhesion level reduces. The best estimates are achieved overall are for the front bogie rear wheelset creep forces. The estimator can also be shown to track adhesion changes in real time, Figure 5. This Figure demonstrates a simple interpolation scheme of the adhesion conditions in the simulation model, and how the estimator successfully track the changes to the creep force level. This is the early stages of this investigation and further work will look into how these estimates are affected by removing some of the measured signals. 


\begin{tabular}{|c|c|c|c|}
\hline Parameter & Description & Value & Units \\
\hline$f_{y 1}$ & $\begin{array}{l}\text { primary lateral damper } \\
\mathrm{CoE}\end{array}$ & 0 & $\mathrm{Ns} / \mathrm{m}$ \\
\hline$f_{\psi 1}$ & $\begin{array}{l}\text { primary yaw damper } \\
\text { CoE }\end{array}$ & 0 & Nms \\
\hline$f_{y 2}$ & $\begin{array}{ll}\text { secondary } & \text { lateral } \\
\text { damper } \mathrm{CoE} & \\
\end{array}$ & $0.06 e^{6}$ & $\mathrm{Ns} / \mathrm{m}$ \\
\hline$I_{b}$ & bogie yaw inertia & 3500 & $\mathrm{kgm}^{2}$ \\
\hline$I_{v}$ & vehicle yaw inertia & 30000 & $\mathrm{kgm}^{2}$ \\
\hline$I_{w}$ & wheelset yaw inertia & 700 & $\mathrm{kgm}^{2}$ \\
\hline$k_{y 1}$ & $\begin{array}{l}\text { primary lateral stiff- } \\
\text { ness }\end{array}$ & $40 e^{6}$ & $\mathrm{~N} / \mathrm{m}$ \\
\hline$k_{y 2}$ & $\begin{array}{l}\text { secondary lateral stiff- } \\
\text { ness }\end{array}$ & $0.1 e^{6}$ & $\mathrm{~N} / \mathrm{m}$ \\
\hline$k_{\psi 1}$ & primary yaw stiffness & $2.5 e^{6}$ & $\mathrm{Nm}$ \\
\hline$m_{b}$ & bogie mass & 2500 & $\mathrm{~kg}$ \\
\hline$m_{v}$ & vehicle mass & 1250 & $\mathrm{~kg}$ \\
\hline$m_{w}$ & wheelset mass & 22000 & $\mathrm{~kg}$ \\
\hline$y_{F F}$ & $\begin{array}{l}\text { front bogie, front } \\
\text { wheelset displacement }\end{array}$ & - & $\mathrm{m}$ \\
\hline$y_{F R}$ & $\begin{array}{l}\text { front bogie, rear } \\
\text { wheelset displacement }\end{array}$ & - & $\mathrm{m}$ \\
\hline$y_{R F}$ & $\begin{array}{l}\text { rear bogie, front } \\
\text { wheelset displacement }\end{array}$ & - & $\mathrm{m}$ \\
\hline$y_{R R}$ & $\begin{array}{l}\text { rear bogie, rear } \\
\text { wheelset displacement }\end{array}$ & - & $\mathrm{m}$ \\
\hline$\psi_{F F}$ & $\begin{array}{l}\text { front bogie, front } \\
\text { wheelset yaw angle }\end{array}$ & - & $\mathrm{rad}$ \\
\hline$\psi_{F R}$ & $\begin{array}{l}\text { front bogie, rear } \\
\text { wheelset yaw angle }\end{array}$ & - & $\mathrm{rad}$ \\
\hline$\psi_{R F}$ & $\begin{array}{l}\text { rear bogie, front } \\
\text { wheelset yaw angle }\end{array}$ & - & $\mathrm{rad}$ \\
\hline$\psi_{R R}$ & $\begin{array}{l}\text { rear bogie, rear } \\
\text { wheelset yaw angle }\end{array}$ & - & $\mathrm{rad}$ \\
\hline
\end{tabular}

Table 2. Model parameters

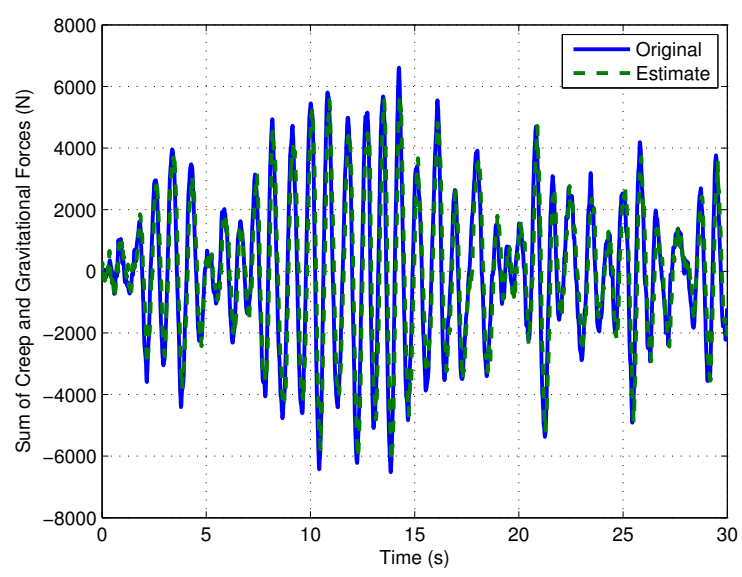

Fig. 4. Estimation of the lateral creep forces

\begin{tabular}{|l|l|l|l|l|}
\hline $\begin{array}{l}\text { Creep } \\
\text { force/ } \\
\text { moment }\end{array}$ & $\begin{array}{l}\text { Dry } \\
R^{2} \\
(\%)\end{array}$ & $\begin{array}{l}\text { Wet } \\
R^{2} \\
(\%)\end{array}$ & $\begin{array}{l}\text { Low } \\
R^{2} \\
(\%)\end{array}$ & $\begin{array}{l}\text { V.Low } \\
R^{2} \\
(\%)\end{array}$ \\
\hline$F_{F F}$ & 83.64 & 82.95 & 69.94 & 65.10 \\
$F_{F R}$ & 85.12 & 84.90 & 79.26 & 73.44 \\
$F_{R F}$ & 85.03 & 84.28 & 73.64 & 71.88 \\
$F_{R R}$ & 85.13 & 85.14 & 72.76 & 65.35 \\
\hline
\end{tabular}

Table 3. Lateral creep forces fit quality
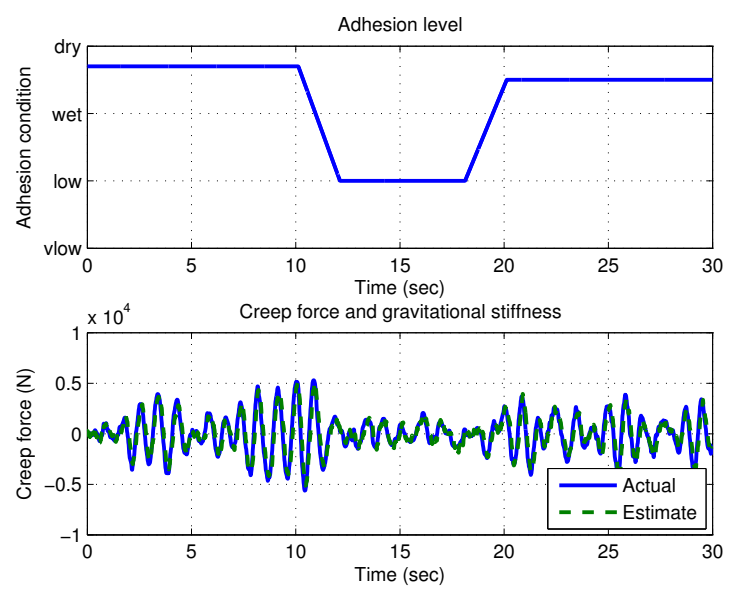

Fig. 5. Varying adhesion estimation

\section{FUTURE WORK}

This study provides a strong theoretical background for a project on on-board detection of low adhesion which started in 2010, administered by the Rail Safety and Strategy Board (RSSB) with funding from the UK's Rail Industry Strategic Research Program. The project has several facets: further development of the data processing algorithms as shown here; how these estimates can be translated into a useful understanding of the adhesion condition; fundamental research into creep characteristics at low creep values using a scale roller rig; and validation of the techniques through data generated by a multi-bodied dynamic simulation package (such as VAMPIRER) and data gathered from a full scale instrumented vehicle.

The intention is also to consider the force estimation technique for many other applications. This could be used for assessing the performance of railway vehicles in terms of the wheel and rail geometric and wear characteristics, perhaps as part of a homologation process. As mentioned earlier this is considered a simpler and cheaper alternative to the use of load measuring wheels which is the current practice. Beyond a homologation process, the technique if applied to a large number, or the majority of the vehicles on the network could be used to help in the prediction wear of the wheel tread, wear of the rail head and it could also be used predict the occurrence of rolling contact fatigue. These latter two points which consider the condition of the infrastructure would require extremely accurate coordination of the position of the vehicle on the network to the forces being estimated, and a study would also have to be generated as to how these forces relate the presence of patterns of wear and fatigue phenomena.

\section{CONCLUSIONS}

This paper discussed development work of the creep force estimation technique around the wheel-rail interface. The primary aim of this research is to develop a real-time system for the estimation of creep forces that could be used to detect local adhesion conditions, and predict the wear generated on the vehicle and the rail infrastructure.

Firstly this paper discussed the development of a full vehicle body non-linear contact mechanics model of the lateral 
and yaw dynamics of the system. This was performed as previous studies highlighted that some of the lateral creep forces and creep moments gave a better estimation than others, and this needed to be investigated in a more representative model of a rail vehicle system.

It then discussed the Kalman-Bucy filter method used to estimate the lateral creep forces and the yaw creep moments of the four wheelset in the model, and how these have given successful estimations in simulations.

Finally the future direction of the project was highlighted with further development potential of the algorithm given, and an explanation of the methods of validation that will be used, by data generated in multi-bodied dynamics simulation software and through track testing. Suggestions were also given for other applications of the creep force estimations.

\section{ACKNOWLEDGEMENTS}

The authors would like to thank Rail Research United Kingdom (RRUK) and the Engineering and Physical Science Research Council (EPSRC) who funded this research.

\section{REFERENCES}

Bombardier (2010). Orbita - predictive asset management, the future of fleet maintenance. http://www.bombardier.com/en/transportation/ accessed 7th April 2010.

Charles, G., Goodall, R., and Dixon, R. (2008). Modelbased condition monitoring at the wheel-rail interface. Vehicle System Dynamics, 46(1), 415-430.

Garg, V. and Dukkipati, R. (1984). Dynamics of Railway Vehicle Systems. Academic Press, first edition.

Grewal, M. and Andrews, A. (2001). Kalman Filtering: Theory and Practice Using MATLAB. WileyInterscience Publications, second edition.

Harrison, H. and McCanney, T. (2002). Recent developments in coefficient of friction measurements at the rail/wheel interface. Wear, 253(1), 114-123.

Hussain, I. and Mei, T. (2010). Multi kalman filtering approach for estimation of wheel-rail contact conditions. In Proceedings of the UKACC control conference, Coventry.

Kalker, J. (1967). On the Rolling Contact of Two Elastic Bodies in the Presence of Dry Friction. Ph.D. thesis, Delft University of Technology, Delft, Netherlands.

Kalman, R. (1960). A new approach to linear filtering and prediction. Transactions of ASME - Journal of Basic Engineering, 35-45.

Li, P., Goodall, R., Weston, P., Ling, C., Goodman, C., and Roberts, C. (2006). Estimation of railway vehicle suspension parameters for condition monitoring. Control Engineering Practice, 15:43-55.

Ljung, A. (1999). System Identification, Theory for the User. Prentice Hall, second edition.

Mei, T. and Li, H. (2008). Measurement of vehicle ground speed using bogie based inertial sensors. IMECHE proceedings, Part F - Rail and Rapid Transit, 222(2), $107-116$.

Pearce, T. and Rose, K. (1985). Measured force-creep relationships and their use in vehicle response calculations. In Proceedings of the IAVSD 9th Symposium, Linkoping.
Polach, O. (2005). Creep forces in simulations of traction vehicles running on adhesion limit. Wear, 258(1), 9921000 .

Vasic, G., Franklin, F., and Kapoor, A. (2003). New rail materials and coatings. Technical Report RRUK/A2/1, University of Sheffield, prepared for RSSB. http://portal.railresearch.org.uk/RRUK/Shared $\% 20$ Documents/rssba2a.pdf.

Ward, C., Goodall, R., and Dixon, R. (2010a). Wheelrail profile condition monitoring. In Proceedings of the UKACC control conference, Coventry.

Ward, C., Weston, P., Stewart, E., Li, H., Goodall, R., Roberts, C., Mei, T., Charles, G., and Dixon, R. (2010b). Condition monitoring opportunities using vehicle based sensors. In press: IMechE proceedings, Part F: Rail and Rapid Transit.

Wickens, A. (2003). Fundamentals of Rail Vehicle Dynamics: Guidance and Stability. Swets and Zeitlinger, first edition.

Xia, F., Cole, C., and Wolfs, P. (2008). Grey box-based inverse wagon model to predict wheel-rail contact forces from measured wagon body responses. Vehicle System Dynamics, 46(Supplement), 469-479. 